Supplemental Information

Multiple TOF/TOF Events in a Single Laser Shot for Improved MALDI MS/MS Quantification Boone M. Prentice, Chad W. Chumbley, Brian C. Hachey, Jeremy L. Norris, and Richard M. Caprioli

\title{
Experimental: Materials
}

Angiotensin II, [glu1]-fibrinopeptide B, enalapril maleate, ramipril, promethazine $\mathrm{HCl}$, verapamil $\mathrm{HCl}$, and trifluoroacetic acid were all purchased from Sigma Aldrich (St. Louis, MO). Enalapril-d5 maleate (phenyl-d5) and verapamil-d3 $\mathrm{HCl}(\mathrm{N}-m e t h y l-\mathrm{d} 3)$ were purchased from C/D/N Isotopes Inc. (Pointe-Claire, Quebec, Canada). Promethazine-d3 HCl was purchased from Cerilliant Corporation (Round Rock, TX, a Sigma Aldrich company). Pooled normal human plasma containing K2EDTA as a coagulant was purchased from Innovative Research (Novi, MI). 2,5-dihydroxybenzoic acid (DHB), methanol, and acetonitrile were purchased from Fisher Scientific (Waltham, MA). 18-M $\Omega$ water was obtained from a Milli-Q water purification system (Millipore, Billerica, MA).

\section{Experimental: Sample Preparation}

For enalapril quantification using ramipril as an internal standard, stock solutions of enalapril maleate $(20.0 \mu \mathrm{M})$ and ramipril $(10.0 \mu \mathrm{M})$ were made in $50 \%$ methanol and stored at $80^{\circ} \mathrm{C}$. The working calibration solutions of enalapril $(0.0300-10.0 \mu \mathrm{M})$ included $0.500 \mu \mathrm{M}$ of ramipril as the internal standard and a final concentration of $45 \%$ pooled human plasma containing K2EDTA as an anticoagulant. Quality control (QC) solutions of $5.00 \mu \mathrm{M}$ and 0.500 $\mu \mathrm{M}$ of enalapril with $0.500-\mu \mathrm{M}$ ramipril in $45 \%$ plasma were also included in the analysis.

For promethazine, enalapril, and verapamil quantification using isotopically labeled internal standards, a stock solution of promethazine hydrochloride, enalapril maleate, and 
verapamil hydrochloride $(20.0 \mu \mathrm{M}$ each $)$ was made in $50 \%$ methanol and stored at $-80^{\circ} \mathrm{C}$. A second stock solution of the isotopically labeled internal standards $(20.0 \mu \mathrm{M}$ each) was also made in $50 \%$ methanol and stored at $-80^{\circ} \mathrm{C}$. The isotopically labeled internal standards were ${ }^{2} \mathrm{H}_{3}$-promethazine hydrochloride (promethazine-d3), ${ }^{2} \mathrm{H}_{5}$-enalapril maleate (enalapril-d5), and ${ }^{2} \mathrm{H}_{3}$-verapamil hydrochloride (verapamil-d3). The working calibration solutions of the drug panel $(0.0300-30.0 \mu \mathrm{M})$ included $5.00 \mu \mathrm{M}$ of internal standards and a final concentration of $45 \%$ pooled human plasma containing K2EDTA as an anticoagulant. Concentrations below the LOQ $(0.700 \mu \mathrm{M})$ have been omitted from the figures for simplicity. The total volume of each solution was $200 \mu \mathrm{L}$. QC solutions of $5.00 \mu \mathrm{M}$ of the drug panel with $5.00 \mu \mathrm{M}$ of the internal standards in $45 \%$ plasma were also included in the analysis.

For both sets of samples, protein precipitation was performed by adding $600 \mu \mathrm{L}$ of acetonitrile to each solution. Following centrifugation at 3,750 RPM for 15 minutes (Sorvall RT Plus, Thermo Scientific), the supernatant for each sample (700 $\mu \mathrm{L})$ was removed and dried down using an SPD Speedvac (Thermo Scientific) at $45^{\circ} \mathrm{C}$ for $\sim 3$ hours. Each sample was then reconstituted in $15.0 \mu \mathrm{L}$ of $50.0 \mathrm{mg} / \mathrm{mL}$ DHB in $50 \%$ methanol with $0.1 \%$ trifluoroacetic acid and the solutions manually spotted $(0.500 \mu \mathrm{L}$ twice for a total volume of $1.000 \mu \mathrm{L})$ onto a goldcoated stainless steel target for analysis. For each set of samples, calibration curves were spotted 7 times and analyzed, with 5 of these included in quantitative calculations based on accuracy (passing QC), spot homogeneity and quality, and successful instrument data acquisition. 

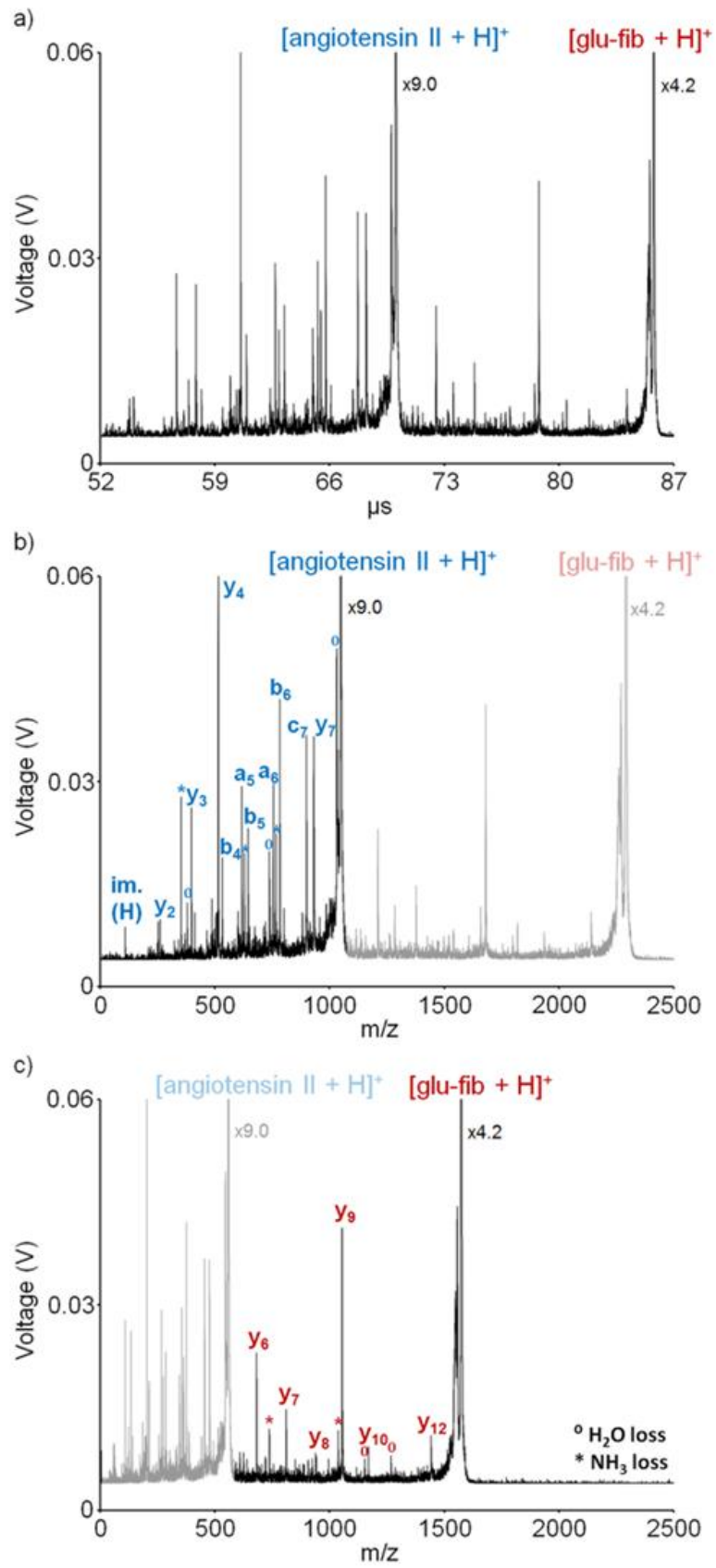

Supplemental Figure 1: a) Two peptide ions, angiotensin II and glu-fib, are each individually fragmented in a single laser shot. Following acquisition, the mass spectrum can then be calibrated using either b) angiotensin II as the precursor ion or c) glu-fib as the precursor ion. The uncalibrated precursor and fragment ions from $b$ and $c$ have been faded. Spectrum represents an average of $\sim 1,000$ laser shots. 

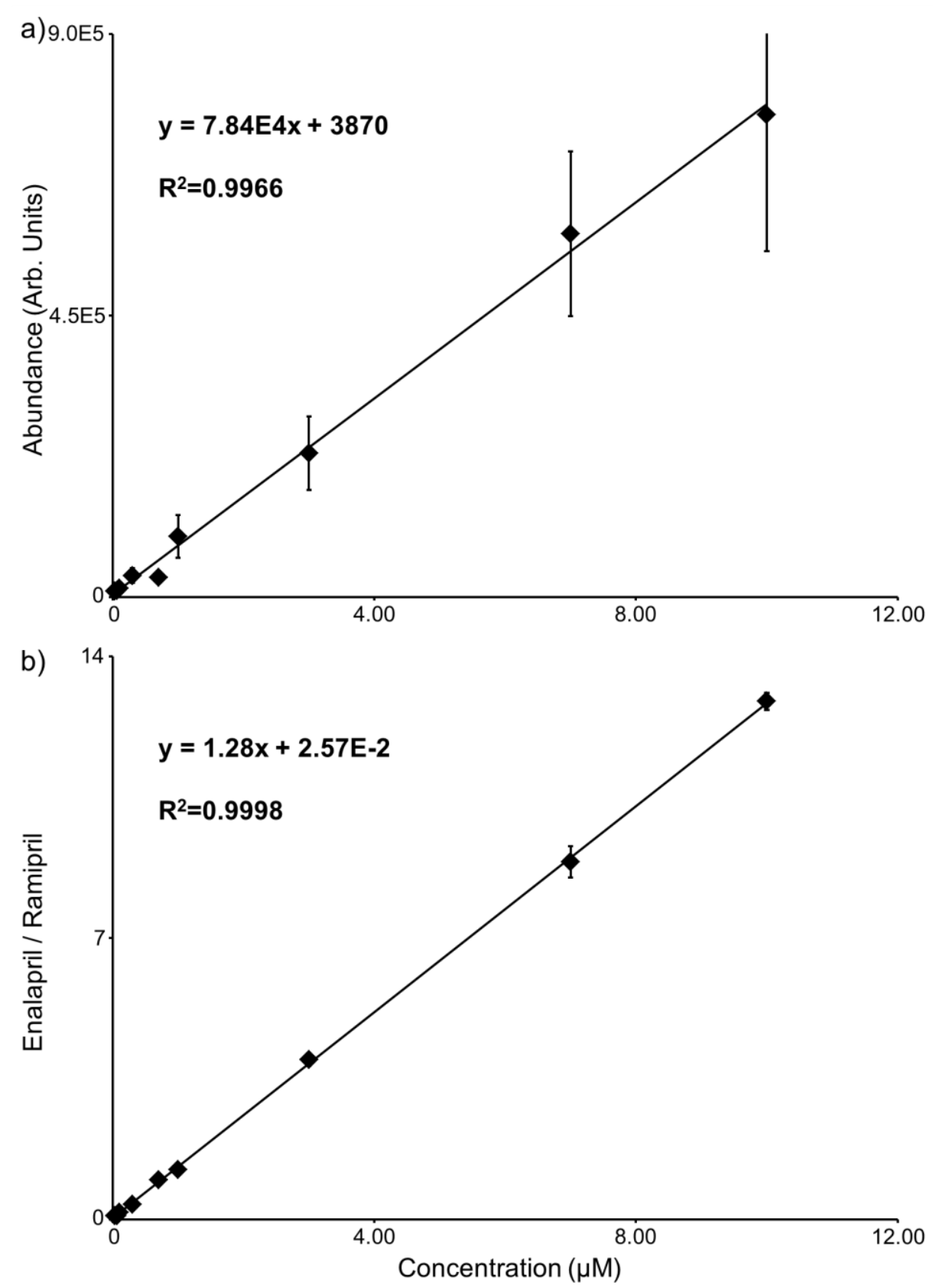

Supplemental Figure 2: Linearity is improved for enalapril quantification from a) without normalization to $b)$ upon normalization to the ramipril internal standard $(n=5)$. 


\begin{tabular}{|c|c|r|r|}
\hline Trial & Quality Control $(\boldsymbol{\mu M})$ & Raw Accuracy (\%) & Ratio Accuracy (\%) \\
\hline 1 & 0.500 & 75.4 & 97.3 \\
\hline & 5.00 & 18.7 & 42.9 \\
\hline 2 & 0.500 & 50.2 & 64.9 \\
\hline & 5.00 & 17.4 & 50.3 \\
\hline 3 & 0.500 & 81.9 & 72.3 \\
\hline & 5.00 & 48.5 & 68.7 \\
\hline 4 & 0.500 & 97.6 & 92.3 \\
\hline & 5.00 & 45.9 & 76.9 \\
\hline 5 & 0.500 & 28.7 & 76.7 \\
\hline & 5.00 & 10.2 & 17.4 \\
\hline & & & 80.7 \\
\hline Average & 0.500 & 66.8 & 51.2 \\
\hline & 5.00 & 28.2 & \\
\hline
\end{tabular}

Supplemental Figure 3: Normalization to the number of spectra acquired at each spot yields only minor improvements in accuracy in enalapril quantification $(n=5)$. 

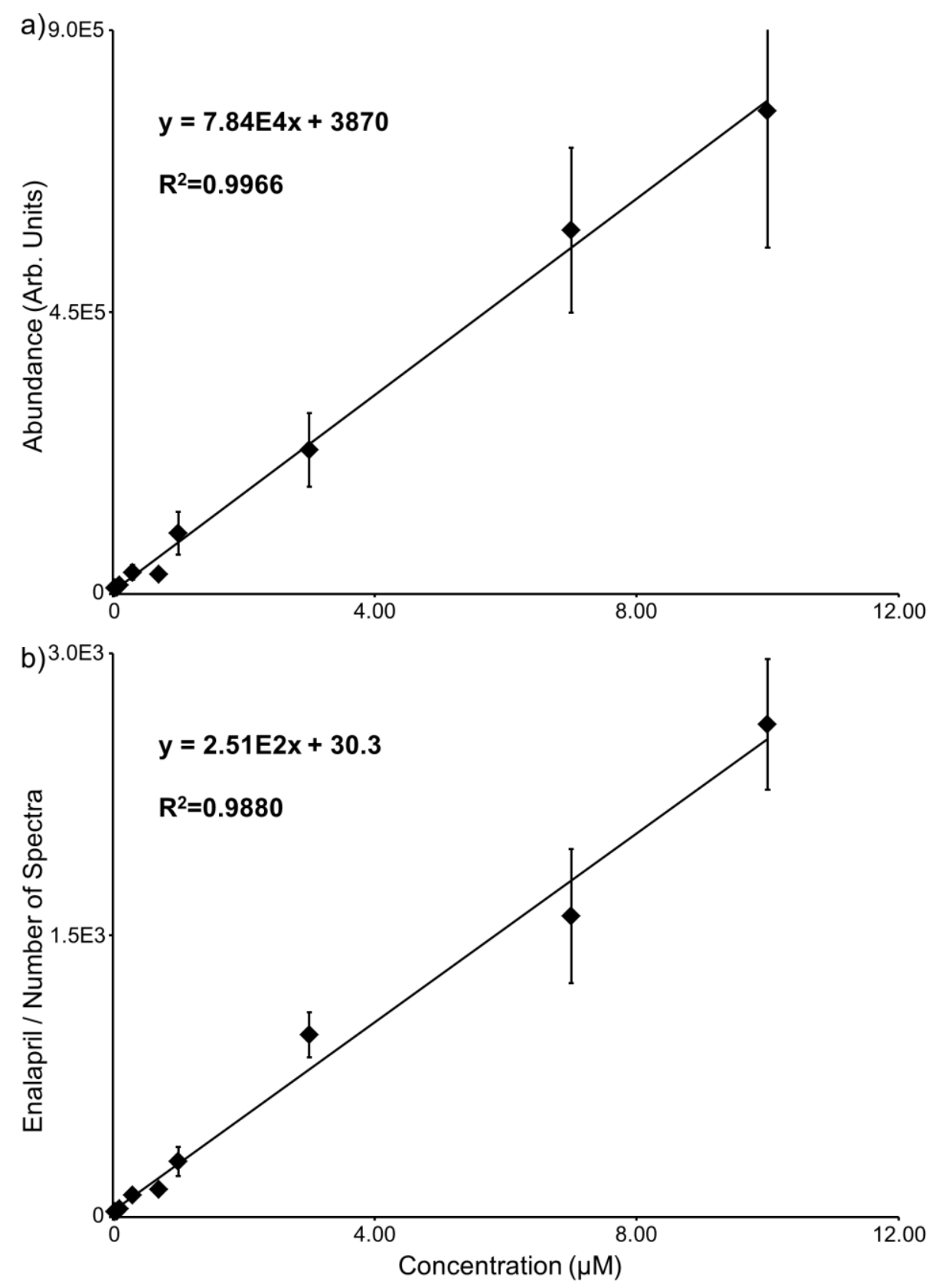

Supplemental Figure 4: Linearity is not improved for enalapril quantification from a) without normalization to $b)$ upon normalization to the number of spectra acquired at each spot $(n=5)$. 


\begin{tabular}{|c|c|c|c|c|}
\hline $\begin{array}{c}\text { Concentration } \\
(\mu \mathrm{M})\end{array}$ & $\begin{array}{l}\text { Average Raw } \\
\text { (Abundance) }\end{array}$ & $\begin{array}{c}\text { Average Ratio } \\
\text { (Enalapril/\#Spectra) }\end{array}$ & $\begin{array}{c}\text { Raw Standard } \\
\text { Deviation (\%) }\end{array}$ & $\begin{array}{c}\text { Ratio Standard } \\
\text { Deviation (\%) }\end{array}$ \\
\hline 0 & 974.94 & 26.598 & 51.02 & 53.20 \\
\hline 0.0300 & $8,733.56$ & 30.275 & 37.57 & 24.13 \\
\hline 0.0700 & $9,949.36$ & 43.446 & 25.18 & 15.51 \\
\hline 0.100 & $13,258.70$ & 116.81 & 37.89 & 27.81 \\
\hline 0.300 & $34,067.25$ & 146.62 & 35.43 & 23.31 \\
\hline 0.700 & $30,910.39$ & 294.50 & 28.00 & 16.89 \\
\hline 1.00 & $96,651.56$ & 969.47 & 35.59 & 26.11 \\
\hline 3.00 & $229,541.16$ & $1,601.2$ & 25.55 & 12.32 \\
\hline 7.00 & $580,569.41$ & $2,621.5$ & 22.63 & 22.33 \\
\hline 10.0 & $771,085.47$ & 26.598 & 28.32 & 13.24 \\
\hline Low QC $(0.500)$ & $31,374.80$ & 153.17 & 54.80 & 35.52 \\
\hline High QC $(5.00)$ & $120,606.44$ & 682.53 & 67.65 & 46.83 \\
\hline
\end{tabular}

Supplemental Figure 5: Normalization to the number of spectra acquired at each spot yields only minor improvements in precision in enalapril quantification $(\mathrm{n}=5)$. 

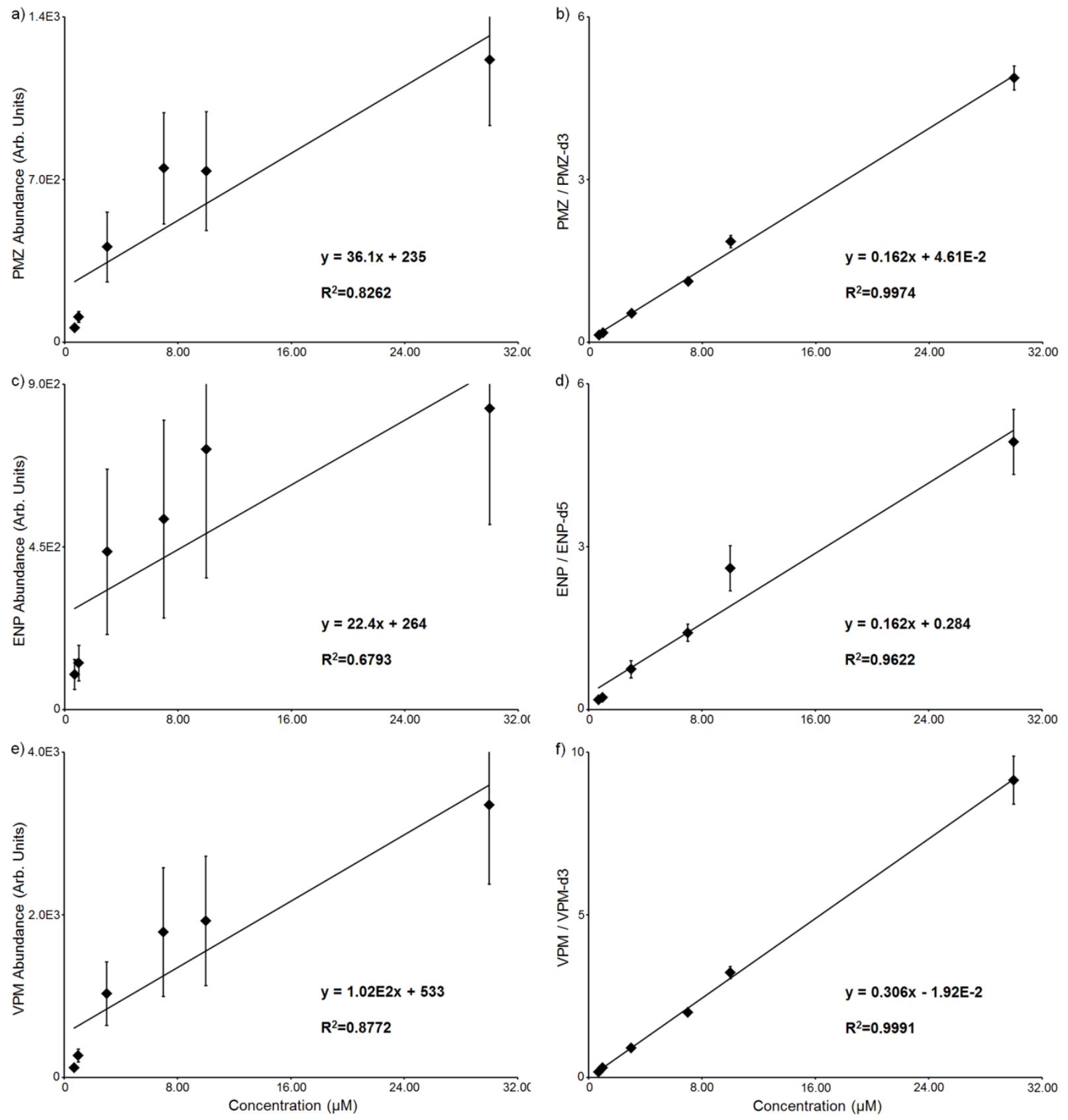

Supplemental Figure 6: Linearity is improved for quantification of a) PMZ upon b) normalization, c) ENP upon d) normalization, and e) VPM upon f) normalization ( $n=5)$. 


\begin{tabular}{|c|c|c|c|c|}
\hline \multirow{7}{*}{$\frac{N}{\Sigma}$} & Trial & Quality Control $(\mu \mathrm{M})$ & Raw Accuracy (\%) & Ratio Accuracy (\%) \\
\hline & 1 & 5.00 & 65.6 & 91.1 \\
\hline & 2 & 5.00 & 51.1 & 80.1 \\
\hline & 3 & 5.00 & 20.0 & 81.4 \\
\hline & 4 & 5.00 & 50.8 & 89.2 \\
\hline & 5 & 5.00 & 63.6 & 82.0 \\
\hline & Average & 5.00 & 50.2 & 84.7 \\
\hline \multirow{7}{*}{ 兄 } & Trial & Quality Control $(\mu \mathrm{M})$ & Raw Accuracy (\%) & Ratio Accuracy (\%) \\
\hline & 1 & 5.00 & 91.1 & 88.6 \\
\hline & 2 & 5.00 & 26.5 & 82.0 \\
\hline & 3 & 5.00 & 48.9 & 74.8 \\
\hline & 4 & 5.00 & 78.7 & 93.0 \\
\hline & 5 & 5.00 & -128 & 86.3 \\
\hline & Average & 5.00 & 23.5 & 85.0 \\
\hline \multirow{7}{*}{$\frac{\Sigma}{2}$} & Trial & Quality Control $(\mu \mathrm{M})$ & Raw Accuracy (\%) & Ratio Accuracy (\%) \\
\hline & 1 & 5.00 & 83.4 & 91.9 \\
\hline & 2 & 5.00 & 44.7 & 86.0 \\
\hline & 3 & 5.00 & 43.2 & 87.0 \\
\hline & 4 & 5.00 & 74.2 & 91.7 \\
\hline & 5 & 5.00 & 29.5 & 90.9 \\
\hline & Average & 5.00 & 55.0 & 89.5 \\
\hline
\end{tabular}

Supplemental Figure 7: Normalization to the isotopically labeled internal standards improves accuracy in in the quantitative analysis of PMZ, ENP, and VPM $(n=5)$. Accuracies are reported as the difference from $100 \%$ of the absolute value of the percent error. 